\title{
GRADED ANNIHILATORS AND UNIFORMLY F-COMPATIBLE IDEALS
}

\author{
RODNEY Y. SHARP
}

\begin{abstract}
Let $R$ be a commutative (Noetherian) local ring of prime characteristic $p$ that is $F$-pure. This paper is concerned with comparison of three finite sets of radical ideals of $R$, one of which is only defined in the case when $R$ is $F$-finite (that is, is finitely generated when viewed as a module over itself via the Frobenius homomorphism). Two of the afore-mentioned three sets have links to tight closure, via test ideals. Among the aims of the paper are a proof that two of the sets are equal, and a proposal for a generalization of I. M. Aberbach's and F. Enescu's splitting prime.
\end{abstract}

\section{Introduction}

Throughout the paper, let $(R, \mathfrak{m})$ be a commutative (Noetherian) local ring of prime characteristic $p$ having maximal ideal $\mathfrak{m}$. In recent years, the study of $R$-modules with a Frobenius action has assisted in the development of the theory of tight closure over $R$. An $R$-module with a Frobenius action can be viewed as a left module over the Frobenius skew polynomial ring over $R$, and such left modules will play a central rôle in this paper.

The Frobenius skew polynomial ring over $R$ is described as follows. Throughout, $f: R \longrightarrow R$ denotes the Frobenius ring homomorphism, for which $f(r)=r^{p}$ for all $r \in R$. The Frobenius skew polynomial ring over $R$ is the skew polynomial ring $R[x, f]$ associated to $R$ and $f$ in the indeterminate $x$; as a left $R$-module, $R[x, f]$ is freely generated by $\left(x^{i}\right)_{i \geq 0}$, and so consists of all polynomials $\sum_{i=0}^{n} r_{i} x^{i}$, where $n \geq 0$ and $r_{0}, \ldots, r_{n} \in R$; however, its multiplication is subject to the rule $x r=f(r) x=r^{p} x$ for all $r \in R$.

We can think of $R[x, f]$ as a positively-graded ring $R[x, f]=\bigoplus_{n=0}^{\infty} R[x, f]_{n}$, where $R[x, f]_{n}=R x^{n}$ for $n \geq 0$. The graded annihilator of a left $R[x, f]$-module $H$ is the largest graded two-sided ideal of $R[x, f]$ that annihilates $H$; it is denoted by gr-ann $_{R[x, f]} H$.

Let $G$ be a left $R[x, f]$-module that is $x$-torsion-free in the sense that $x g=0$, for

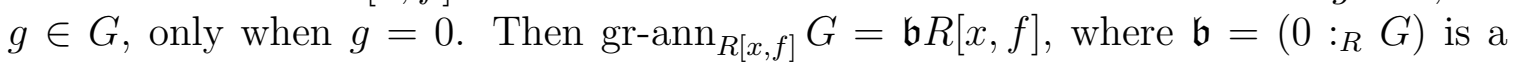
radical ideal. See [11, Lemma 1.9]. We shall use $\mathcal{I}(G)$ (or $\mathcal{I}_{R}(G)$ ) to denote the set of $R$-annihilators of the $R[x, f]$-submodules of $G$; we shall refer to the members of $\mathcal{I}(G)$

Date: June 21, 2018.

2010 Mathematics Subject Classification. Primary 13A35, 16S36, 13E05, 13F40; Secondary 13J10.

Key words and phrases. Commutative Noetherian local ring, prime characteristic, Frobenius homomorphism, tight closure, test element, excellent ring, Frobenius skew polynomial ring, graded annihilator, $F$-pure ring, uniformly $F$-compatible ideal. 
as the $G$-special $R$-ideals. For a graded two-sided ideal $\mathfrak{B}$ of $R[x, f]$, we denote by $\operatorname{ann}_{G}(\mathfrak{B})$ or $\operatorname{ann}_{G} \mathfrak{B}$ the $R[x, f]$-submodule of $G$ consisting of all elements of $G$ that are annihilated by $\mathfrak{B}$. Also, we shall use $\mathcal{A}(G)$ to denote the set of special annihilator submodules of $G$, that is, the set of $R[x, f]$-submodules of $G$ of the form $\operatorname{ann}_{G}(\mathfrak{A})$, where $\mathfrak{A}$ is a graded two-sided ideal of $R[x, f]$. In [11, $\S 1]$, the present author showed that there is a sort of 'Galois' correspondence between $\mathcal{I}(G)$ and $\mathcal{A}(G)$. In more detail, there is an order-reversing bijection, $\Delta: \mathcal{A}(G) \longrightarrow \mathcal{I}(G)$ given by

$$
\Delta: N \longmapsto\left(\text { gr-ann }_{R[x, f]} N\right) \cap R=\left(0:_{R} N\right) .
$$

The inverse bijection, $\Delta^{-1}: \mathcal{I}(G) \longrightarrow \mathcal{A}(G)$, also order-reversing, is given by

$$
\left.\Delta^{-1}: \mathfrak{b} \longmapsto \operatorname{ann}_{G}(\mathfrak{b} R[x, f])\right) \text {. }
$$

We shall be mainly concerned in this paper with the situation where $R$ is $F$-pure. We remind the reader what this means. For $j \in \mathbb{N}$ (the set of positive integers) and an $R$-module $M$, let $M^{(j)}$ denote $M$ considered as a left $R$-module in the natural way and as a right $R$-module via $f^{j}$, the $j$ th iterate of the Frobenius ring homomorphism. Then $R$ is $F$-pure if, for every $R$-module $M$, the natural map $M \longrightarrow R^{(1)} \otimes_{R} M$ (which maps $m \in M$ to $1 \otimes m$ ) is injective.

Note that $R^{(j)} \cong R x^{j}$ as $(R, R)$-bimodules. Let $i \in \mathbb{N}_{0}$, the set of non-negative integers. When we endow $R x^{i}$ and $R x^{j}$ with their natural structures as $(R, R)$ bimodules (inherited from their being graded components of $R[x, f]$ ), there is an isomorphism of (left) $R$-modules $\phi: R x^{i+j} \otimes_{R} M \stackrel{\cong}{\longrightarrow} R x^{i} \otimes_{R}\left(R x^{j} \otimes_{R} M\right)$ for which $\phi\left(r x^{i+j} \otimes m\right)=r x^{i} \otimes\left(x^{j} \otimes m\right)$ for all $r \in R$ and $m \in M$. It follows that $R$ is $F$-pure if and only if the left $R[x, f]$-module $R[x, f] \otimes_{R} M$ is $x$-torsion-free for every $R$-module $M$. This means that, when $R$ is $F$-pure, there is a good supply of natural $x$-torsion-free left $R[x, f]$-modules.

In fact, we shall use $\Phi$ (or $\Phi_{R}$ when it is desirable to specify which ring is being considered) to denote the functor $R[x, f] \otimes_{R}$ - from the category of $R$-modules (and all $R$-homomorphisms) to the category of all $\mathbb{N}_{0}$-graded left $R[x, f]$-modules (and all homogeneous $R[x, f]$-homomorphisms). For an $R$-module $M$, we shall identify $\Phi(M)$ with $\bigoplus_{n \in \mathbb{N}_{0}} R x^{n} \otimes_{R} M$, and (usually) identify its 0th component $R \otimes_{R} M$ with $M$, in the obvious ways.

Let $E$ be the injective envelope of the simple $R$-module $R / \mathfrak{m}$. We shall be concerned with $\Phi(E)$, the $\mathbb{N}_{0}$-graded left $R[x, f]$-module $\bigoplus_{n \in \mathbb{N}_{0}} R x^{n} \otimes_{R} E$. Assume now that $R$ is $F$-pure. In [12, Corollary 4.11], the present author proved that the set $\mathcal{I}(\Phi(E))$ is a finite set of radical ideals of $R$; in [11, Theorem 3.6 and Corollary 3.7], he proved that $\mathcal{I}(\Phi(E))$ is closed under taking primary (prime in this case) components; and in [14, Corollary 2.8], he proved that the big test ideal $\widetilde{\tau}(R)$ of $R$ (for tight closure) is equal to the smallest member of $\mathcal{I}(\Phi(E))$ that meets $R^{\circ}$, the complement in $R$ of the union of the minimal prime ideals of $R$.

Let $\mathfrak{a} \in \mathcal{I}(\Phi(E))$ (with $\mathfrak{a} \neq R$ ), still in the $F$-pure case. The special annihilator submodule $\operatorname{ann}_{\Phi(E)}(\mathfrak{a} R[x, f])$ of $\Phi(E)$ corresponding to $\mathfrak{a}$ inherits a natural structure as a graded left module over the Frobenius skew polynomial ring $(R / \mathfrak{a})[x, f]$, and its 0 th component is contained in $\left(0:_{E} \mathfrak{a}\right)$. As $R / \mathfrak{a}$-module, the latter is isomorphic to the injective envelope of the simple $R / \mathfrak{a}$-module. Motivated by results in [14, §3] in the case where $R$ is complete, and by work of K. Schwede in [10, §5] in the F-finite 
case, we say that $\mathfrak{a}$ is fully $\Phi(E)$-special if (it is $\Phi(E)$-special and) its 0th component is exactly $\left(0:_{E} \mathfrak{a}\right)$. The main result of this paper is that a $\Phi(E)$-special ideal of $R$ is always fully $\Phi(E)$-special provided that $R$ is an (F-pure) homomorphic image of an excellent regular local ring of characteristic $p$. When $R$ satisfies this condition, corollaries can be drawn from that main result: we shall establish an analogue of [14, Theorem 3.1] and, in particular, show that $R / \mathfrak{a}$ is $F$-pure whenever $\mathfrak{a}$ is a proper $\Phi(E)$-special ideal of $R$.

Along the way, we shall show that, in the case where $R$ is $F$-finite as well as $F$ pure, the set $\mathcal{I}(\Phi(E))$ of $\Phi(E)$-special ideals of $R$ is equal to the set of uniformly $F$-compatible ideals of $R$, introduced by K. Schwede in [10, §3]. An ideal $\mathfrak{b}$ of $R$ is said to be uniformly $F$-compatible if, for every $j>0$ and every $\phi \in \operatorname{Hom}_{R}\left(R^{(j)}, R\right)$, we have $\phi\left(\mathfrak{b}^{(j)}\right) \subseteq \mathfrak{b}$. In [10, Corollary 5.3 and Corollary 3.3], Schwede proved that there are only finitely many uniformly $F$-compatible ideals of $R$ and that they are all radical; in [10, Proposition 4.7 and Corollary 4.8], he proved that the set of uniformly $F$-compatible ideals is closed under taking primary (prime in this case) components; in [10, Theorem 6.3], Schwede proved that the big test ideal $\widetilde{\tau}(R)$ of $R$ is equal to the smallest uniformly $F$-compatible ideal of $R$ that meets $R^{\circ}$; and in [10, Remark 4.4 and Proposition 4.7], he proved that there is a unique largest proper uniformly $F$-compatible ideal of $R$, and that that is prime and equal to the splitting prime of $R$ discovered and defined by I. M. Aberbach and F. Enescu [1, §3].

Thus, in the $F$-finite $F$-pure case, the set of uniformly $F$-compatible ideals of $R$ has properties similar to some properties of $\mathcal{I}(\Phi(E))$. Are the two sets the same? We shall, during the course of the paper, show that the answer is 'yes'. It should be emphasized, however, that Schwede only defined uniformly $F$-compatible ideals in the $F$-finite case, whereas the majority of this paper is devoted to the study of fully $\Phi(E)$-special ideals in the ( $F$-pure but) not necessarily $F$-finite case.

We shall use the notation of this Introduction throughout the remainder of the paper. In particular, $R$ will denote a local ring of prime characteristic $p$ having maximal ideal $\mathfrak{m}$. We shall sometimes use the notation $(R, \mathfrak{m})$ just to remind the reader that $R$ is local. The completion of $R$ will be denoted by $\widehat{R}$. We shall only assume that $R$ is reduced, or $F$-pure, or $F$-finite, when there is an explicit statement to that effect; also $E$ will continue to denote $E_{R}(R / \mathfrak{m})$. We continue to use $\mathbb{N}$, respectively $\mathbb{N}_{0}$, to denote the set of all positive, respectively non-negative, integers.

For $j \in \mathbb{N}_{0}$, the $j$ th component of an $\mathbb{N}_{0}$-graded left $R[x, f]$-module $G$ will be denoted by $G_{j}$.

\section{Fully $\Phi(E)$-SPECIAL IDEALS}

We remind the reader that we usually identify the 0th component of $\Phi(E)=$ $\bigoplus_{n \in \mathbb{N}_{0}} R x^{n} \otimes_{R} E$ with $E$ in the obvious natural way. For an ideal $\mathfrak{a}$ of $R$, we have, with this convention, that the 0 th component of $\operatorname{ann}_{\Phi(E)}(\mathfrak{a} R[x, f])$ is contained in $\left(0:_{E} \mathfrak{a}\right)$.

1.1. Lemma. Assume that $(R, \mathfrak{m})$ is F-pure; let $\mathfrak{a}$ be an ideal of $R$. Then the 0 th component $\left(\operatorname{ann}_{\Phi(E)}(\mathfrak{a} R[x, f])\right)_{0}$ of $\operatorname{ann}_{\Phi(E)}(\mathfrak{a} R[x, f])$ contains $\left(0:_{E} \mathfrak{a}\right)$ if and only if $\mathfrak{a}$ is $\Phi(E)$-special and $\left(\operatorname{ann}_{\Phi(E)}(\mathfrak{a} R[x, f])\right)_{0}=\left(0:_{E} \mathfrak{a}\right)$.

Proof. Only the implication ' $\Rightarrow$ ' needs proof. 
Assume that $\left(0:_{E} \mathfrak{a}\right) \subseteq\left(\operatorname{ann}_{\Phi(E)}(\mathfrak{a} R[x, f])\right)_{0}$. Since $\operatorname{ann}_{\Phi(E)}(\mathfrak{a} R[x, f])$ is an $R[x, f]$ submodule of $\Phi(E)$, it follows that $\operatorname{ann}_{\Phi(E)}(\mathfrak{a} R[x, f])$ contains the image $J$ of the map

$$
\Phi\left(\left(0:_{E} \mathfrak{a}\right)\right)=R[x, f] \otimes_{R}\left(0:_{E} \mathfrak{a}\right) \longrightarrow R[x, f] \otimes_{R} E=\Phi(E)
$$

induced by inclusion. Let $\mathfrak{b}$ be the radical ideal of $R$ for which $\operatorname{gr}^{-a n n n_{R[x, f]} J=}$ $\mathfrak{b} R[x, f]$, so that $\mathfrak{b}=(0: R J)$. As $J \subseteq \operatorname{ann}_{\Phi(E)}(\mathfrak{a} R[x, f])$, we must have $\mathfrak{a} \subseteq \mathfrak{b}$. Furthermore, $\mathfrak{b}$ annihilates $\left(0:_{E} \mathfrak{a}\right) \cong \operatorname{Hom}_{R}(R / \mathfrak{a}, E)$, and since an $R$-module and its Matlis dual have the same annihilator, we also have $\mathfrak{b} \subseteq \mathfrak{a}$. Thus $\mathfrak{a}=\mathfrak{b}$ is the $R$-annihilator of an $R[x, f]$-submodule of $\Phi(E)$, and so $\mathfrak{a} \in \mathcal{I}(\Phi(E))$.

Finally, note that an $e \in\left(\operatorname{ann}_{\Phi(E)}(\mathfrak{a} R[x, f])\right)_{0}$ must be annihilated by $\mathfrak{a}$, and so lies in $\left(0:_{E} \mathfrak{a}\right)$.

1.2. Definition. Assume that $(R, \mathfrak{m})$ is $F$-pure; let $\mathfrak{a}$ be an ideal of $R$. We say that $\mathfrak{a}$ is fully $\Phi(E)$-special if the equivalent conditions of Lemma 1.1 are satisfied.

Thus $\mathfrak{a}$ is fully $\Phi(E)$-special if and only if $\left(0:_{E} \mathfrak{a}\right) \subseteq\left(\operatorname{ann}_{\Phi(E)}(\mathfrak{a} R[x, f])\right)_{0}$, and, then, $\mathfrak{a}$ is $\Phi(E)$-special and we have the equality $\left(0:_{E} \mathfrak{a}\right)=\left(\operatorname{ann}_{\Phi(E)}(\mathfrak{a} R[x, f])\right)_{0}$.

To facilitate the presentation of some examples of $\Phi(E)$-special ideals that are fully $\Phi(E)$-special, we review next the theory of $S$-tight closure, where $S$ is a multiplicatively closed subset of $R$. This theory was developed in [14]. The special case of the theory in which $S=R^{\circ}$ is the 'classical' tight closure theory of M. Hochster and C. Huneke [2].

1.3. Reminders. Let $H$ be a left $R[x, f]$-module and let $S$ be a multiplicatively closed subset of $R$.

(i) We define the internal $S$-tight closure of zero in $H$, denoted $\Delta^{S}(H)$, to be the $R[x, f]$-submodule of $H$ given by

$\Delta^{S}(H)=\left\{h \in H\right.$ : there exists $s \in S$ with $s x^{n} h=0$ for all $\left.n \gg 0\right\}$.

When $M$ is an $R$-module and we take the graded left $R[x, f]$-module $\Phi(M)=$ $R[x, f] \otimes_{R} M$ for $H$, the $R[x, f]$-submodule $\Delta^{S}(\Phi(M))$ of $\Phi(M)$ is graded, and we refer to its 0 th component as the $S$-tight closure of 0 in $M$, or the tight closure with respect to $S$ of 0 in $M$, and denote it by $0_{M}^{*, S}$. See [14, $\left.\S 1\right]$.

(ii) By [14, Example 1.3(ii)], we have, for an $R$-module $M$,

$$
\Delta^{S}\left(R[x, f] \otimes_{R} M\right)=0_{M}^{*, S} \oplus 0_{R x \otimes_{R} M}^{*, S} \oplus \cdots \oplus 0_{R x^{n} \otimes_{R} M}^{*, S} \oplus \cdots .
$$

(iii) Recall that an $S$-test element for $R$ is an element $s \in S$ such that, for every $R$-module $M$ and every $j \in \mathbb{N}_{0}$, the element $s x^{j}$ annihilates $1 \otimes m \in(\Phi(M))_{0}$ for every $m \in 0_{M}^{*, S}$. The ideal of $R$ generated by all the $S$-test elements for $R$ is called the $S$-test ideal of $R$, and denoted by $\tau^{S}(R)$.

1.4. Reminders. Suppose that $(R, \mathfrak{m})$ is $F$-pure. Let $S$ be a multiplicatively closed subset of $R$. Recall that the set $\mathcal{I}(\Phi(E))$ of $\Phi(E)$-special $R$-ideals is finite; let $\mathfrak{b}^{S, \Phi(E)}$ denote the intersection of all the minimal members of the set

$$
\{\mathfrak{p} \in \operatorname{Spec}(R) \cap \mathcal{I}(\Phi(E)): \mathfrak{p} \cap S \neq \emptyset\} .
$$

Thus $\mathfrak{b}^{S, \Phi(E)}$ is the smallest member of $\mathcal{I}(\Phi(E))$ that meets $S$. 
(i) By [14, Theorem 2.6], the set $S \cap \mathfrak{b}^{S, \Phi(E)}$ is (non-empty and) equal to the set of $S$-test elements for $R$.

(ii) Thus there exists an $S$-test element for $R$.

(iii) Furthermore, $\Delta^{S}(\Phi(E))=\operatorname{ann}_{\Phi(E)}\left(\mathfrak{b}^{S, \Phi(E)} R[x, f]\right)$ and $\left(0:_{R} \Delta^{S}(\Phi(E))\right)=$ $\mathfrak{b}^{S, \Phi(E)}$, by [14, Proposition 1.5].

(iv) By [14, Proposition 2.10(v)], we have $\mathfrak{b}^{S, \Phi(E)}=\left(0:_{R} 0_{E}^{*, S}\right)$.

1.5. Lemma (Sharp [14, Corollary 2.8]). Suppose that $(R, \mathfrak{m})$ is F-pure. Let $S$ be the complement in $R$ of the union of finitely many prime ideals.

Then the $S$-test ideal $\tau^{S}(R)$ is equal to $\mathfrak{b}^{S, \Phi(E)}$, the smallest member of the finite set $\mathcal{I}(\Phi(E))$ that meets $S$.

We shall also use the following result from [14].

1.6. Theorem (Sharp [14, Theorem 2.12]). Suppose that $(R, \mathfrak{m})$ is F-pure. Let $\mathfrak{a} \in$ $\mathcal{I}(\Phi(E))$. Then there exists a multiplicatively closed subset $S$ of $R$ such that $\mathfrak{a}$ is the $S$-test ideal of $R$. Moreover, $S$ can be taken to be the complement in $R$ of the union of finitely many prime ideals.

We are now able to give examples of fully $\Phi(E)$-special ideals, because the next result shows that, when $(R, \mathfrak{m})$ is complete and $F$-pure, a $\Phi(E)$-special ideal of $R$ is automatically fully $\Phi(E)$-special.

1.7. Proposition. Suppose that $(R, \mathfrak{m})$ is complete and F-pure. Then every $\Phi(E)$ special ideal of $R$ is fully $\Phi(E)$-special.

Proof. Let $\mathfrak{a}$ be a $\Phi(E)$-special ideal of $R$. If $\mathfrak{a}=R$, then

$$
\left(0:_{E} \mathfrak{a}\right)=0 \subseteq \operatorname{ann}_{\Phi(E)}(\mathfrak{a} R[x, f])
$$

and $\mathfrak{a}$ is fully $\Phi(E)$-special. We therefore assume that $\mathfrak{a}$ is proper.

By Theorem [1.6 and [14, Corollary 2.8], there exist finitely many prime ideals $\mathfrak{p}_{1}, \ldots, \mathfrak{p}_{n}$ of $R$ such that, if we set $S:=R \backslash \bigcup_{i=1}^{n} \mathfrak{p}_{i}$, then $\mathfrak{a}$ is the $S$-test ideal of $R$, that is $\mathfrak{a}=\tau^{S}(R)=\mathfrak{b}^{S, \Phi(E)}$, where the notation is as in 1.3)(iii) and 1.4. Therefore, by 1.3(ii) and 1.4(iii),

$$
\begin{aligned}
0_{E}^{*, S} \oplus 0_{R x \otimes_{R} E}^{*, S} \oplus \cdots \oplus 0_{R x^{n} \otimes_{R} E}^{*, S} \oplus \cdots & =\Delta^{S}(\Phi(E)) \\
& =\operatorname{ann}_{\Phi(E)}\left(\mathfrak{b}^{S, \Phi(E)} R[x, f]\right) .
\end{aligned}
$$

Now we know that $\mathfrak{b}^{S, \Phi(E)}=\left(0:_{R} 0_{E}^{*, S}\right)$, by 1.4(iv). Since $R$ is complete, it follows from Matlis duality (see, for example, [15, p. 154]) that $0_{E}^{*, S}=\left(0:_{E} \mathfrak{b}^{S, \Phi(E)}\right)$. We have thus shown that $\left(0:_{E} \mathfrak{a}\right)=R \otimes_{R}\left(0:_{E} \mathfrak{a}\right) \subseteq\left(\operatorname{ann}_{\Phi(E)}(\mathfrak{a} R[x, f])\right)_{0}$. Thus $\mathfrak{a}$ is fully $\Phi(E)$-special.

Next, we develop some theory for fully $\Phi(E)$-special ideals.

1.8. Lemma. Suppose that $(R, \mathfrak{m})$ is F-pure, and let $\mathfrak{a}$ be a fully $\Phi(E)$-special ideal of $R$. Then $\mathfrak{a}$ is radical and every associated prime of $\mathfrak{a}$ is also fully $\Phi(E)$-special.

Proof. We can assume that $\mathfrak{a}$ is proper. Since $\mathfrak{a}$ is $\Phi(E)$-special, it must be radical. Let $\mathfrak{a}=\mathfrak{p}_{1} \cap \cdots \cap \mathfrak{p}_{t}$ be the minimal primary (prime in this case) decomposition of $\mathfrak{a}$, and let $i \in\{1, \ldots, t\}$. 
Since $\mathfrak{a}$ is fully $\Phi(E)$-special, we have $\left(0:_{E} \mathfrak{a}\right) \subseteq\left(\operatorname{ann}_{\Phi(E)}(\mathfrak{a} R[x, f])\right)_{0}$. Let $e \in$ $\left(0:_{E} \mathfrak{p}_{i}\right)$ and let $r \in \mathfrak{p}_{i}$. We show that $r x^{n}$ annihilates the element $1 \otimes e$ of the 0 th component of $\Phi(E)$. There exists

$$
a \in \bigcap_{\substack{j=1 \\ j \neq i}}^{t} \mathfrak{p}_{j} \backslash \mathfrak{p}_{i} .
$$

Now $\left(0:_{E} \mathfrak{p}_{i}\right)=a\left(0:_{E} \mathfrak{p}_{i}\right)$, because multiplication by a provides a monomorphism of $R / \mathfrak{p}_{i}$ into itself and $E$ is injective. Therefore $e=a e^{\prime}$ for some $e^{\prime} \in\left(0:_{E} \mathfrak{p}_{i}\right)$. Therefore $r x^{n} \otimes e=r x^{n} \otimes a e^{\prime}=r a^{p^{n}} x^{n} \otimes e^{\prime}=0$ since $r a^{p^{n}} \in \mathfrak{a}$ and

$$
\left(0:_{E} \mathfrak{p}_{i}\right) \subseteq\left(0:_{E} \mathfrak{a}\right) \subseteq \operatorname{ann}_{\Phi(E)}(\mathfrak{a} R[x, f]) .
$$

Therefore $\left(0:_{E} \mathfrak{p}_{i}\right) \subseteq\left(\operatorname{ann}_{\Phi(E)}\left(\mathfrak{p}_{i} R[x, f]\right)\right)_{0}$ and $\mathfrak{p}_{i}$ is fully $\Phi(E)$-special.

1.9. Proposition. Suppose that $(R, \mathfrak{m})$ is F-pure. Let $\left(\mathfrak{a}_{\lambda}\right)_{\lambda \in \Lambda}$ be a non-empty family of fully $\Phi(E)$-special ideals of $R$. Then $\sum_{\lambda \in \Lambda} \mathfrak{a}_{\lambda}$ is again fully $\Phi(E)$-special.

Proof. Set $\mathfrak{a}:=\sum_{\lambda \in \Lambda} \mathfrak{a}_{\lambda}$, and observe that $\mathfrak{a} R[x, f]=\sum_{\lambda \in \Lambda}\left(\mathfrak{a}_{\lambda} R[x, f]\right)$. By assumption, we have $\left(0:_{E} \mathfrak{a}_{\lambda}\right) \subseteq \operatorname{ann}_{\Phi(E)}\left(\mathfrak{a}_{\lambda} R[x, f]\right)$ for all $\lambda \in \Lambda$. It follows that

$$
\begin{aligned}
\left(0:_{E} \mathfrak{a}\right) & =\left(0:_{E} \sum_{\lambda \in \Lambda} \mathfrak{a}_{\lambda}\right)=\bigcap_{\lambda \in \Lambda}\left(0:_{E} \mathfrak{a}_{\lambda}\right) \\
& \subseteq \bigcap_{\lambda \in \Lambda}\left(\operatorname{ann}_{\Phi(E)}\left(\mathfrak{a}_{\lambda} R[x, f]\right)\right)_{0} \\
& =\left(\operatorname{ann}_{\Phi(E)}\left(\sum_{\lambda \in \Lambda}\left(\mathfrak{a}_{\lambda} R[x, f]\right)\right)\right)_{0}=\left(\operatorname{ann}_{\Phi(E)}(\mathfrak{a} R[x, f])\right)_{0} .
\end{aligned}
$$

Therefore $\mathfrak{a}:=\sum_{\lambda \in \Lambda} \mathfrak{a}_{\lambda}$ is fully $\Phi(E)$-special.

1.10. Corollary. Suppose that $(R, \mathfrak{m})$ is $F$-pure. Then $R$ has a unique largest fully $\Phi(E)$-special proper ideal, and this is prime.

Proof. The zero ideal is fully $\Phi(E)$-special, and so it follows from Proposition 1.9 that the sum $\mathfrak{b}$ of all the fully $\Phi(E)$-special proper ideals of $R$ is fully $\Phi(E)$-special (and contained in $\mathfrak{m}$ ), and so is the unique largest fully $\Phi(E)$-special proper ideal of $R$. Also $\mathfrak{b}$ must be prime, since all the associated primes of $\mathfrak{b}$ are fully $\Phi(E)$-special, by Lemma 1.8.

In what follows, we shall have cause to pass between $R$ and its completion. Note that if $R$ is $F$-pure, then so too is $\widehat{R}$, by Hochster and Roberts [3, Corollary 6.13]. The following technical lemma will be helpful.

1.11. Lemma. (See [13, Lemma 4.3].) There is a unique way of extending the $R$ module structure on $E:=E_{R}(R / \mathfrak{m})$ to an $\widehat{R}$-module structure. Recall that, as an $\widehat{R}$-module, $E \cong E_{\widehat{R}}(\widehat{R} / \widehat{\mathfrak{m}})$.

Since each element of $\Phi_{R}(E)=R[x, f] \otimes_{R} E$ is annihilated by some power of $\mathfrak{m}$, the left $R[x, f]$-module structure on $\Phi_{R}(E)$ can be extended in a unique way to a left $\widehat{R}[x, f]$-module structure.

The $\operatorname{map} \beta: \Phi_{R}(E)=R[x, f] \otimes_{R} E \longrightarrow \widehat{R}[x, f] \otimes_{\widehat{R}} E=\Phi_{\widehat{R}}(E)$ for which

$$
\beta\left(r x^{i} \otimes h\right)=r x^{i} \otimes h \quad \text { for all } r \in R, i \in \mathbb{N}_{0} \text { and } h \in E
$$

is a homogeneous $\widehat{R}[x, f]$-isomorphism. 
Since each element of $\Phi_{R}(E)$ is annihilated by some power of $\mathfrak{m}$, it follows that a subset of $\Phi_{R}(E)$ is an $R[x, f]$-submodule if and only if it is an $\widehat{R}[x, f]$-submodule. Consequently,

$$
\mathcal{I}_{R}\left(\Phi_{R}(E)\right)=\left\{\mathfrak{B} \cap R: \mathfrak{B} \in \mathcal{I}_{\widehat{R}}\left(\Phi_{\widehat{R}}(E)\right)\right\} .
$$

1.12. Lemma. Suppose that $(R, \mathfrak{m})$ is F-pure, and let $\mathfrak{a}$ be an ideal of $R$. Then $\mathfrak{a} \widehat{R}$ is a fully $\Phi_{\widehat{R}}(E)$-special ideal of $\widehat{R}$ if and only if $\mathfrak{a}$ is a fully $\Phi_{R}(E)$-special ideal of $R$.

Proof. By Lemma 1.11, when we extend the left $R[x, f]$-module structure on $\Phi_{R}(E)$, in the unique way possible, to a left $\widehat{R}[x, f]$-module structure, $E \cong E_{\widehat{R}}(\widehat{R} / \widehat{\mathfrak{m}})$ as $\widehat{R}$ modules and $\Phi_{R}(E) \cong \Phi_{\widehat{R}}(E)$ as left $\widehat{R}[x, f]$-modules. The claim therefore follows from the facts that

$$
\operatorname{ann}_{\Phi_{R}(E)}(\mathfrak{a} R[x, f])=\operatorname{ann}_{\Phi_{R}(E)}((\mathfrak{a} \widehat{R}) \widehat{R}[x, f])
$$

and $\left(0:_{E} \mathfrak{a}\right)=\left(0:_{E} \mathfrak{a} \widehat{R}\right)$.

2. The case where $R$ is an F-pure homomorphic image of an excellent regular local ring of characteristic $p$

The main aim of this section is to prove that, when $R$ is an $F$-pure homomorphic image of an excellent regular local ring of characteristic $p$, every $\Phi(E)$-special ideal of $R$ is fully $\Phi(E)$-special ideal. This will enable us to extend some results obtained in [14, §3] about an $F$-pure complete local ring to an $F$-pure homomorphic image of an excellent regular local ring of characteristic $p$. We begin the section with a lemma that is derived from a result of G. Lyubeznik [5, Lemma 4.1].

2.1. Lemma. Let $(S, \mathfrak{M})$ be a complete regular local ring of characteristic $p$, and let $\mathfrak{B}$ be a proper, non-zero ideal of $S$. Denote $E_{S}(S / \mathfrak{M})$ by $E_{S}$, and let $S[x, f]$ denote the Frobenius skew polynomial ring over $S$. Let $n \in \mathbb{N}$.

Since $S$ is regular, $S^{(n)}$ is faithfully flat over $S$, and we identify $S x^{n} \otimes_{S}\left(0:_{E_{S}} \mathfrak{B}\right)$ as an $S$-submodule of $S x^{n} \otimes_{S} E_{S}$ in the natural way. Let $a_{1}, \ldots, a_{d}$ be a regular system of parameters for $S$. Consider the $S$-isomorphism $\delta_{n}: S x^{n} \otimes_{S} E_{S} \stackrel{\cong}{\longrightarrow} E_{S}$ of [11, 4.2(iii)], for which (with the notation used in the statement of that result)

$$
\delta_{n}\left(b x^{n} \otimes\left[\frac{s}{\left(a_{1} \ldots a_{d}\right)^{j}}\right]\right)=\left[\frac{b s^{p^{n}}}{\left(a_{1} \ldots a_{d}\right)^{j p^{n}}}\right] \quad \text { for all } b, s \in S \text { and } j \in \mathbb{N}_{0} .
$$

The isomorphism $\delta_{n}$ maps

(i) $S x^{n} \otimes_{S}\left(0:_{E_{S}} \mathfrak{B}\right)$ onto $\left(0:_{E_{S}} \mathfrak{B}^{\left[p^{n}\right]}\right)$, and

(ii) $\mathfrak{B}\left(S x^{n} \otimes_{S}\left(0:_{E_{S}} \mathfrak{B}\right)\right)$ onto $\left(0:_{E_{S}}\left(\mathfrak{B}^{\left[p^{n}\right]}: \mathfrak{B}\right)\right)$.

Proof. (i) Use of the analogue of Lyubeznik [5, Lemma 4.1] for the functor $S x^{n} \otimes_{S}$ • shows that the Matlis dual of $S x^{n} \otimes_{S}\left(0: E_{S} \mathfrak{B}\right)$ is $S$-isomorphic to $S x^{n} \otimes_{S}(S / \mathfrak{B}) \cong$ $S / \mathfrak{B}^{\left[p^{n}\right]}$. Since each $S$-module has the same annihilator as its Matlis dual, we thus see that $S x^{n} \otimes_{S}\left(0::_{E_{S}} \mathfrak{B}\right)$ has annihilator $\mathfrak{B}^{\left[p^{n}\right]}$. Since $S$ is complete, we have $T=\left(0:_{E_{S}}\left(0:_{S} T\right)\right)$ for each submodule $T$ of $E_{S}$, by Matlis duality (see, for example, [15, p. 154]). It therefore follows that

$$
\delta_{n}\left(S x^{n} \otimes_{S}\left(0:_{E_{S}} \mathfrak{B}\right)\right)=\left(0:_{E_{S}} \mathfrak{B}^{\left[p^{n}\right]}\right) .
$$


(ii) Set $N:=S x^{n} \otimes_{S}\left(0:_{E_{S}} \mathfrak{B}\right)$. Similar reasoning shows that

$$
\delta_{n}(\mathfrak{B} N)=\left(0:_{E_{S}}\left(0:_{S} \mathfrak{B} N\right)\right)=\left(0:_{E_{S}}\left(\left(0:_{S} N\right): \mathfrak{B}\right)\right)=\left(0:_{E_{S}}\left(\mathfrak{B}^{\left[p^{n}\right]}: \mathfrak{B}\right)\right)
$$

2.2. Proposition. Suppose that $R=S / \mathfrak{A}$, where $(S, \mathfrak{M})$ is a regular local ring of characteristic $p$, and $\mathfrak{A}$ is a proper ideal of $S$. Assume also that $R$ is $F$-pure. Let $\mathfrak{b}$ be a proper ideal of $R$; let $\mathfrak{B}$ be the unique ideal of $S$ that contains $\mathfrak{A}$ and is such that $\mathfrak{B} / \mathfrak{A}=\mathfrak{b}$.

Then $\mathfrak{b}$ is fully $\Phi(E)$-special if and only if $\left(\mathfrak{A}^{\left[p^{n}\right]}: \mathfrak{A}\right) \subseteq\left(\mathfrak{B}^{\left[p^{n}\right]}: \mathfrak{B}\right)$ for all $n \in \mathbb{N}$.

Note. In the F-finite case, this result is already known and due to K. Schwede [10, Proposition 3.11 and Lemma 5.1].

Proof. If $\mathfrak{A}=0$, then $R$ is regular, so that its big test ideal is $R$ itself (by [6, Theorem 8.8], for example) and the only proper $\Phi(E)$-special ideal of $R$ is 0 ; also, $\left(0^{\left[p^{n}\right]}: 0\right)=S$, and the only proper ideal $\mathfrak{B}$ of $S$ satisfying $\left(0^{\left[p^{n}\right]}: 0\right) \subseteq\left(\mathfrak{B}^{\left[p^{n}\right]}: \mathfrak{B}\right)$ for all $n \in \mathbb{N}$ is the zero ideal. Thus the result is true when $\mathfrak{A}=0$; we therefore assume for the remainder of this proof that $\mathfrak{A} \neq 0$.

Note that $\widehat{R}=\widehat{S} / \mathfrak{A} \widehat{S}$ is again $F$-pure and that $\widehat{S}$ is an excellent complete regular local ring of characteristic $p$, with maximal ideal $\mathfrak{M} \widehat{S}$.

We also note that $\mathfrak{b}$ is a fully $\Phi_{R}(E)$-special ideal of $R$ if and only if $\mathfrak{b} \widehat{R}$ is a fully $\Phi_{\widehat{R}}(E)$-special ideal of $\widehat{R}$, by Lemma 1.12. Furthermore, by the faithful flatness of $\widehat{S}$ over $S$, we have, for $n \in \mathbb{N}$,

$$
\left.\left((\mathfrak{A} \widehat{S})^{\left[p^{n}\right]}: \mathfrak{A} \widehat{S}\right)=\left(\mathfrak{A}^{\left[p^{n}\right]}: \mathfrak{A}\right) \widehat{S} \subseteq\left(\mathfrak{B}^{\left[p^{n}\right]}: \mathfrak{B}\right) \widehat{S}=(\mathfrak{B} \widehat{S})^{\left[p^{n}\right]}: \mathfrak{B} \widehat{S}\right)
$$

if and only if $\left(\mathfrak{A}^{\left[p^{n}\right]}: \mathfrak{A}\right) \subseteq\left(\mathfrak{B}^{\left[p^{n}\right]}: \mathfrak{B}\right)$. Therefore, we can, and do, assume henceforth in this proof that $S$ is complete.

Let $E_{S}:=E_{S}(S / \mathfrak{M})$. Now $\left(0:_{E_{S}} \mathfrak{A}\right)=E:=E_{R}(R / \mathfrak{m})$ and $\left(0:_{E_{S}} \mathfrak{B}\right)=\left(0:_{E} \mathfrak{b}\right)$. Note that $\mathfrak{b}$ is fully $\Phi_{R}(E)$-special if and only if, for each $n \in \mathbb{N}$ and each $r \in \mathfrak{b}$, the element $r x^{n} \in R x^{n}$ annihilates the $R$-submodule $\left(0:_{E} \mathfrak{b}\right)$ of the 0 th component $E$ of $\Phi_{R}(E)$.

Let $n \in \mathbb{N}$. There is an exact sequence of $(S, S)$-bimodules

$$
0 \longrightarrow \mathfrak{A} S x^{n} \stackrel{\subseteq}{\longrightarrow} S x^{n} \stackrel{\nu}{\longrightarrow} R x^{n} \longrightarrow 0,
$$

where $\nu\left(s x^{n}\right)=(s+\mathfrak{A}) x^{n}$ for all $s \in S$. The map

$$
S x^{n} \otimes_{S}\left(0:_{E_{S}} \mathfrak{A}\right) \longrightarrow R x^{n} \otimes_{S}\left(0:_{E_{S}} \mathfrak{A}\right)=R x^{n} \otimes_{R}\left(0:_{E_{S}} \mathfrak{A}\right)=R x^{n} \otimes_{R} E
$$

induced by $\nu$ therefore has kernel $\mathfrak{A}\left(S x^{n} \otimes_{S}\left(0:_{E_{S}} \mathfrak{A}\right)\right)$.

It follows that $\mathfrak{b}$ is fully $\Phi_{R}(E)$-special if and only if, for all $n \in \mathbb{N}, s \in \mathfrak{B}$ and $g \in\left(0:_{E_{S}} \mathfrak{B}\right)=\left(0:_{E} \mathfrak{b}\right)$, the element $s x^{n} \otimes g$ of $S x^{n} \otimes_{S}\left(0:_{E_{S}} \mathfrak{A}\right)$ lies in

$$
\mathfrak{A}\left(S x^{n} \otimes_{S}\left(0: E_{S} \mathfrak{A}\right)\right) .
$$

In other words, $\mathfrak{b}$ is fully $\Phi_{R}(E)$-special if and only if, for all $n \in \mathbb{N}$, we have

$$
\mathfrak{B}\left(S x^{n} \otimes_{S}\left(0:_{E_{S}} \mathfrak{B}\right)\right) \subseteq \mathfrak{A}\left(S x^{n} \otimes_{S}\left(0: E_{S} \mathfrak{A}\right)\right) .
$$

(We are here identifying $S x^{n} \otimes_{S}\left(0:_{E_{S}} \mathfrak{B}\right)$ and $S x^{n} \otimes_{S}\left(0:_{E_{S}} \mathfrak{A}\right)$ with submodules of $S x^{n} \otimes_{S} E_{S}$ in the obvious ways, using the faithful flatness of $S^{(n)}$ over $S$.) 
By [11, 4.2(iii)], we have $S x^{n} \otimes_{S} E_{S} \cong E_{S}$. Since $S$ is complete, each submodule $T$ of $E_{S}$ satisfies $T=\left(0:_{E_{S}}\left(0:_{S} T\right)\right)$. Set $N:=S x^{n} \otimes_{S} E_{S}$. Thus

$$
\mathfrak{A}\left(S x^{n} \otimes_{S}\left(0:_{E_{S}} \mathfrak{A}\right)\right)=\left(0:_{N}\left(0:_{S}\left(\mathfrak{A}\left(S x^{n} \otimes_{S}\left(0:_{E_{S}} \mathfrak{A}\right)\right)\right)\right)\right)=\left(0:_{N}\left(\mathfrak{A}^{\left[p^{n}\right]}: \mathfrak{A}\right)\right),
$$

by Lemma 2.1. Similarly, $\mathfrak{B}\left(S x^{n} \otimes_{S}\left(0:_{E_{S}} \mathfrak{B}\right)\right)=\left(0:_{N}\left(\mathfrak{B}^{\left[p^{n}\right]}: \mathfrak{B}\right)\right)$. It follows that $\mathfrak{b}$ is fully $\Phi_{R}(E)$-special if and only if

$$
\left(0:_{N}\left(\mathfrak{B}^{\left[p^{n}\right]}: \mathfrak{B}\right)\right) \subseteq\left(0:_{N}\left(\mathfrak{A}^{\left[p^{n}\right]}: \mathfrak{A}\right)\right) \text { for all } n \in \mathbb{N},
$$

that is (since $\left.N \cong E_{S}\right)$, if and only if $\left(\mathfrak{A}^{\left[p^{n}\right]}: \mathfrak{A}\right) \subseteq\left(\mathfrak{B}^{\left[p^{n}\right]}: \mathfrak{B}\right)$ for all $n \in \mathbb{N}$.

2.3. Theorem. Suppose that $R=S / \mathfrak{A}$ is a homomorphic image of an excellent regular local ring $(S, \mathfrak{M})$ of characteristic $p$, modulo a proper ideal $\mathfrak{A}$. Assume that $R$ is $F$ pure.

Then each $\Phi(E)$-special ideal of $R$ is fully $\Phi(E)$-special.

Proof. Once again, the claim is easy to prove if $\mathfrak{A}=0$, and so we assume henceforth in this proof that $\mathfrak{A} \neq 0$

Note that $\widehat{R}=\widehat{S} / \mathfrak{A} \widehat{S}$ is again $F$-pure and that $\widehat{S}$ is an excellent complete regular local ring of characteristic $p$, with maximal ideal $\mathfrak{M} \widehat{S}$.

Let $\mathfrak{b}$ be a $\Phi(E)$-special $R$-ideal with $\mathfrak{b} \neq R$. Then $\mathfrak{b}=\mathfrak{c} \cap R$ for some $\Phi_{\widehat{R}}(E)$ special $\widehat{R}$-ideal $\mathfrak{c}$. (We have used Lemma 1.11 here.) Let $\mathfrak{C}$ be the unique ideal of $\widehat{S}$ that contains $\mathfrak{A} \widehat{S}$ and is such that $\mathfrak{C} / \mathfrak{A} \widehat{S}=\mathfrak{c}$. By Proposition 1.7, the ideal $\mathfrak{c}$ of $\widehat{R}$ is fully $\Phi_{\widehat{R}}(E)$-special, and so, by Proposition 2.2 , we have

$$
\left(\mathfrak{A}^{\left[p^{n}\right]}: \mathfrak{A}\right) \widehat{S}=\left((\mathfrak{A} \widehat{S})^{\left[p^{n}\right]}: \mathfrak{A} \widehat{S}\right) \subseteq\left(\mathfrak{C}^{\left[p^{n}\right]}: \mathfrak{C}\right) \quad \text { for all } n \in \mathbb{N} .
$$

Set $\mathfrak{C} \cap S:=\mathfrak{B}$, so that $\mathfrak{B} / \mathfrak{A}=\mathfrak{b}$.

Let $n \in \mathbb{N}$ and $s \in\left(\mathfrak{A}^{\left[p^{n}\right]}: S \mathfrak{A}\right)$. Therefore $s \in\left(\mathfrak{C}^{\left[p^{n}\right]}: \mathfrak{C}\right)$. It follows from G. Lyubeznik and K. E. Smith [6, Lemma 6.6] that $\mathfrak{C}^{\left[p^{n}\right]} \cap S=(\mathfrak{C} \cap S)^{\left[p^{n}\right]}$. (Lyubeznik's and Smith's proof of this result uses work of N. Radu [9, Corollary 5], which, in turn, uses D. Popescu's general Néron desingularization [7, 8].) We can now deduce that

$$
s(\mathfrak{C} \cap S) \subseteq s \mathfrak{C} \cap S \subseteq \mathfrak{C}^{\left[p^{n}\right]} \cap S=(\mathfrak{C} \cap S)^{\left[p^{n}\right]},
$$

so that $s \in\left((\mathfrak{C} \cap S)^{\left[p^{n}\right]}: \mathfrak{C} \cap S\right)=\left(\mathfrak{B}^{\left[p^{n}\right]}: \mathfrak{B}\right)$.

We have thus shown that $\left(\mathfrak{A}^{\left[p^{n}\right]}: \mathfrak{A}\right) \subseteq\left(\mathfrak{B}^{\left[p^{n}\right]}: \mathfrak{B}\right)$ for all $n \in \mathbb{N}$, so that $\mathfrak{b}=\mathfrak{B} / \mathfrak{A}$ is fully $\Phi(E)$-special by Proposition 2.2 .

In the case where $R$ is an $F$-pure homomorphic image of an excellent regular local ring of characteristic $p$, the characterization of $\mathcal{I}(\Phi(E))$ afforded by Proposition 2.2 and Theorem 2.3 enables us to see that that set behaves well under localization. As the ideals in $\mathcal{I}(\Phi(E))$ are precisely those that can be expressed as intersections of finitely many prime members of $\mathcal{I}(\Phi(E))$, it is of interest to examine the behaviour of $\mathcal{I}(\Phi(E)) \cap \operatorname{Spec}(R)$ under localization. The next proposition, which is an extension of part of [12, Proposition 2.8], is in preparation for this investigation.

2.4. Proposition. Let $S$ be a regular local ring of characteristic $p$, and let $n \in \mathbb{N}$. Let $\mathfrak{A}, \mathfrak{B}_{1}, \ldots, \mathfrak{B}_{t}, \mathfrak{C}$ be ideals of $S$ with $0 \neq \mathfrak{A} \neq S$, and let $\mathfrak{A}=\mathfrak{Q}_{1} \cap \ldots \cap \mathfrak{Q}_{t}$ be a minimal primary decomposition of $\mathfrak{A}$.

(i) We have $\left(\mathfrak{B}_{1} \cap \cdots \cap \mathfrak{B}_{t}\right)^{\left[p^{n}\right]}=\mathfrak{B}_{1}^{\left[p^{n}\right]} \cap \cdots \cap \mathfrak{B}_{t}^{\left[p^{n}\right]}$. 
(ii) If $\mathfrak{Q}$ is a $\mathfrak{P}$-primary ideal of $S$, then $\mathfrak{Q}^{\left[p^{n}\right]}$ is also $\mathfrak{P}$-primary.

(iii) The equation $\mathfrak{A}^{\left[p^{n}\right]}=\mathfrak{Q}_{1}^{\left[p^{n}\right]} \cap \cdots \cap \mathfrak{Q}_{t}^{\left[p^{n}\right]}$ provides a minimal primary decomposition of $\mathfrak{A}^{\left[p^{n}\right]}$.

(iv) We have $(\mathfrak{A}: \mathfrak{C})^{\left[p^{n}\right]}=\left(\mathfrak{A}^{\left[p^{n}\right]}: \mathfrak{C}^{\left[p^{n}\right]}\right)$ and $\left(\mathfrak{A}^{\left[p^{n}\right]}: \mathfrak{A}\right) \subseteq\left((\mathfrak{A}: \mathfrak{C})^{\left[p^{n}\right]}:(\mathfrak{A}: \mathfrak{C})\right)$.

(v) If $\mathfrak{P}$ is an associated prime ideal of $\mathfrak{A}$, then $\left(\mathfrak{A}^{\left[p^{n}\right]}: \mathfrak{A}\right) \subseteq\left(\mathfrak{P}^{\left[p^{n}\right]}: \mathfrak{P}\right)$.

(vi) Since $0 \neq \mathfrak{A} \neq S$, we have $\left(\mathfrak{A}^{\left[p^{n}\right]}: \mathfrak{A}\right) \neq S$. If $\mathfrak{P}_{1}:=\sqrt{\mathfrak{Q}_{1}}$ is a minimal prime ideal of $\mathfrak{A}$, then $\mathfrak{P}_{1}$ is a minimal prime ideal of $\left(\mathfrak{A}^{\left[p^{n}\right]}: \mathfrak{A}\right)$ and the unique $\mathfrak{P}_{1}$-primary component of $\left(\mathfrak{A}^{\left[p^{n}\right]}: \mathfrak{A}\right)$ is $\left(\mathfrak{Q}_{1}^{\left[p^{n}\right]}: \mathfrak{Q}_{1}\right)$.

Proof. Parts (i), (ii) and (iii) were essentially proved in [12, Proposition 2.8], while parts (iv), (v) and (vi) can be proved by obvious modifications of the arguments used to prove the corresponding parts of [12, Proposition 2.8].

2.5. Corollary. Suppose that $R$ is F-pure and a homomorphic image of an excellent regular local ring $S$ of characteristic $p$ modulo a proper ideal $\mathfrak{A}$. Let $\mathfrak{p} \in \operatorname{Spec}(R)$. Then

$$
\mathcal{I}_{R_{\mathfrak{p}}}\left(\Phi_{R_{\mathfrak{p}}}\left(E_{R_{\mathfrak{p}}}\left(R_{\mathfrak{p}} / \mathfrak{p} R_{\mathfrak{p}}\right)\right)\right) \cap \operatorname{Spec}\left(R_{\mathfrak{p}}\right)=\left\{\mathfrak{q} R_{\mathfrak{p}}: \mathfrak{q} \in \mathcal{I}(\Phi(E)) \cap \operatorname{Spec}(R) \text { and } \mathfrak{q} \subseteq \mathfrak{p}\right\}
$$

Proof. Note that, by M. Hochster and J. L. Roberts [3, Lemma 6.2], the localization $R_{\mathfrak{p}}$ is again $F$-pure. The claim is easy to prove when $\mathfrak{A}=0$, and so we assume that $\mathfrak{A} \neq 0$.

For each lower case fraktur letter that denotes an ideal of $R$, let the corresponding upper case fraktur letter denote the unique ideal of $S$ that contains $\mathfrak{A}$ and has quotient modulo $\mathfrak{A}$ equal to the specified ideal of $R$. For example, $\mathfrak{P}$ denotes the unique ideal of $S$ that contains $\mathfrak{A}$ and is such that $\mathfrak{P} / \mathfrak{A}=\mathfrak{p}$.

Note that $R_{\mathfrak{p}} \cong S_{\mathfrak{P}} / \mathfrak{A} S_{\mathfrak{P}}$ is again a homomorphic image of an excellent regular local ring $S$ of characteristic $p$. Let $\mathfrak{q} \in \operatorname{Spec}(R)$ with $\mathfrak{q} \subseteq \mathfrak{p}$.

Suppose first that $\mathfrak{q} \in \mathcal{I}(\Phi(E)) \cap \operatorname{Spec}(R)$. By Theorem 2.3, we see that $\mathfrak{q}$ is fully $\Phi(E)$-special; use of Proposition $\left[2.2\right.$ shows that $\left(\mathfrak{A}^{\left[p^{n}\right]}: \mathfrak{A}\right) \subseteq\left(\mathfrak{Q}^{\left[p^{n}\right]}: \mathfrak{Q}\right)$ for all $n \in \mathbb{N}$. Therefore

$$
\left(\left(\mathfrak{A} S_{\mathfrak{P}}\right)^{\left[p^{n}\right]}: \mathfrak{A} S_{\mathfrak{P}}\right) \subseteq\left(\left(\mathfrak{Q} S_{\mathfrak{P}}\right)^{\left[p^{n}\right]}: \mathfrak{Q} S_{\mathfrak{P}}\right) \text { for all } n \in \mathbb{N} \text {. }
$$

Since the standard isomorphism $S_{\mathfrak{P}} / \mathfrak{A} S_{\mathfrak{P}} \cong R_{\mathfrak{p}}$ maps $\mathfrak{Q} S_{\mathfrak{P}} / \mathfrak{A} S_{\mathfrak{P}}$ onto $\mathfrak{q} R_{\mathfrak{p}}$, it follows from Proposition 2.2 that $\mathfrak{q} R_{\mathfrak{p}}$ is fully $\Phi_{R_{\mathfrak{p}}}\left(E_{R_{\mathfrak{p}}}\left(R_{\mathfrak{p}} / \mathfrak{p} R_{\mathfrak{p}}\right)\right)$-special.

Conversely, suppose that $\mathfrak{q} R_{\mathfrak{p}}$ is $\Phi_{R_{\mathfrak{p}}}\left(E_{R_{\mathfrak{p}}}\left(R_{\mathfrak{p}} / \mathfrak{p} R_{\mathfrak{p}}\right)\right)$-special, so that, by Theorem 2.3, it is fully $\Phi_{R_{\mathfrak{p}}}\left(E_{R_{\mathfrak{p}}}\left(R_{\mathfrak{p}} / \mathfrak{p} R_{\mathfrak{p}}\right)\right)$-special. By Proposition 2.2, this means that

$$
\left(\left(\mathfrak{A} S_{\mathfrak{P}}\right)^{\left[p^{n}\right]}: \mathfrak{A} S_{\mathfrak{P}}\right) \subseteq\left(\left(\mathfrak{Q} S_{\mathfrak{P}}\right)^{\left[p^{n}\right]}: \mathfrak{Q} S_{\mathfrak{P}}\right) \quad \text { for all } n \in \mathbb{N} \text {. }
$$

Let ${ }^{e}$ and ${ }^{c}$ denote extension and contraction of ideals under the natural ring homomorphism $S \longrightarrow S_{\mathfrak{P}}$. Contract the last displayed inclusion relations back to $S$ to see that

$$
\left(\mathfrak{A}^{\left[p^{n}\right]}: \mathfrak{A}\right) \subseteq\left(\mathfrak{A}^{\left[p^{n}\right]}: \mathfrak{A}\right)^{e c} \subseteq\left(\mathfrak{Q}^{\left[p^{n}\right]}: \mathfrak{Q}\right)^{e c}=\left(\mathfrak{Q}^{\left[p^{n}\right]}: \mathfrak{Q}\right) \text { for all } n \in \mathbb{N}
$$

because $\left(\mathfrak{Q}^{\left[p^{n}\right]}: \mathfrak{Q}\right)$ is $\mathfrak{Q}$-primary (for all $n \in \mathbb{N}$ ), by Proposition 2.4(vi). It follows from Proposition 2.2 that $\mathfrak{Q} / \mathfrak{A}=\mathfrak{q}$ is fully $\Phi(E)$-special.

We can now recover a special case of a result of Lyubeznik and Smith. 
2.6. Corollary (G. Lyubeznik and K. E. Smith [6, Theorem 7.1]). Suppose that $R$ is $F$-pure and a homomorphic image of an excellent regular local ring $S$ of characteristic $p$ modulo a proper ideal $\mathfrak{A}$. Let $\mathfrak{p} \in \operatorname{Spec}(R)$. Then the big test ideal of $R_{\mathfrak{p}}$ is the extension to $R_{\mathfrak{p}}$ of the big test ideal of $R$. In symbols, $\widetilde{\tau}\left(R_{\mathfrak{p}}\right)=\widetilde{\tau}(R) R_{\mathfrak{p}}$.

Proof. The big test ideal $\widetilde{\tau}(R)$ of $R$ is equal to the intersection of the (finitely many) members of $\mathcal{I}(\Phi(E)) \cap \operatorname{Spec}(R)$ of positive height, and a similar statement holds for $R_{\mathfrak{p}}$. The claim therefore follows from Corollary 2.5,

Some results were obtained in [14, Theorem 3.1] for an F-pure complete local ring of characteristic $p$. We can now use Theorem 2.3 to establish analogous results for an $F$-pure homomorphic image of an excellent regular local ring of characteristic $p$.

2.7. Theorem. Suppose $(R, \mathfrak{m})$ is $F$-pure and that every $\Phi(E)$-special ideal of $R$ is fully $\Phi(E)$-special. (For example, by Theorem 2.3, this would be the case if $R$ were a homomorphic image of an excellent regular local ring of characteristic $p$.) Let $\mathfrak{c}$ be a proper ideal of $R$ that is $\Phi(E)$-special. In the light of Theorem 1.6 , let $\mathfrak{p}_{1}, \ldots, \mathfrak{p}_{w}$ be prime ideals of $R$ for which the multiplicatively closed subset $S=R \backslash \bigcup_{i=1}^{w} \mathfrak{p}_{i}$ of $R$ satisfies $\mathfrak{c}=\tau^{S}(R)$. Set $J:=\Delta^{S}(\Phi(E))$, a graded left $R[x, f]$-module.

(i) We have $J=0_{E}^{*, S} \oplus 0_{R x \otimes_{R} E}^{*, S} \oplus \cdots \oplus 0_{R x^{n} \otimes_{R} E}^{*, S} \oplus \cdots=\operatorname{ann}_{\Phi(E)}(\mathfrak{c} R[x, f])$.

(ii) When we regard $J$ as a graded left $(R / \mathfrak{c})[x, f]$-module in the natural way, it is $x$-torsion-free and has $\mathcal{I}_{R / \mathfrak{c}}(J)=\{\mathfrak{g} / \mathfrak{c}: \mathfrak{g} \in \mathcal{I}(\Phi(E)): \mathfrak{g} \supseteq \mathfrak{c}\}$.

(iii) The 0 th component $J_{0}$ of $J$ is $\left(0:_{E} \mathfrak{c}\right)$; as $R / \mathfrak{c}$-module, this is isomorphic to $E_{R / \mathfrak{c}}((R / \mathfrak{c}) /(\mathfrak{m} / \mathfrak{c}))$.

(iv) The ring $R / \mathfrak{c}$ is $F$-pure.

(v) We have $\mathcal{I}\left(\Phi_{R / \mathfrak{c}}\left(J_{0}\right)\right) \subseteq \mathcal{I}_{R / \mathfrak{c}}(J)$, so that

$\left\{\mathfrak{d}: \mathfrak{d}\right.$ is an ideal of $R$ with $\mathfrak{d} \supseteq \mathfrak{c}$ and $\left.\mathfrak{d} / \mathfrak{c} \in \mathcal{I}\left(\Phi_{R / \mathfrak{c}}\left(J_{0}\right)\right)\right\} \subseteq \mathcal{I}\left(\Phi_{R}(E)\right)$.

Proof. Since the $\Phi(E)$-special ideal $\mathfrak{c}$ is fully $\Phi(E)$-special, we have $J_{0}=\left(\begin{array}{ll}0 & :_{E} \\ \mathfrak{c}\end{array}\right)$. Given this observation, one can now use the arguments employed in the proof of [14, Theorem 3.1] to furnish a proof of this theorem.

The next corollary follows from Theorem 2.7 just as, in [14, Corollary 3.2 follows from Theorem 3.1.

2.8. Corollary. Suppose that $(R, \mathfrak{m})$ is local, $F$-pure and that every $\Phi(E)$-special ideal of $R$ is fully $\Phi(E)$-special. (For example, by Theorem 2.3, this would be the case if $R$ were a homomorphic image of an excellent regular local ring of characteristic $p$.) Let $\mathfrak{c}$ be a proper ideal of $R$ that is $\Phi(E)$-special. Denote $R / \mathfrak{c}$ by $\bar{R}$, and note that $\bar{R}$ is F-pure, by Theorem 2.7(iv). Let $T$ be a multiplicatively closed subset of $\bar{R}$ which is the complement in $\bar{R}$ of the union of finitely many prime ideals. The finitistic $T$-test ideal $\tau^{\mathrm{fg}, T}(\bar{R})$ of $\bar{R}$ is defined to be $\bigcap_{L}\left(0: \bar{R} 0_{L}^{*, T}\right)$, where the intersection is taken over all finitely generated $\bar{R}$-modules $L$.

(i) If $\mathfrak{h}$ denotes the unique ideal of $R$ that contains $\mathfrak{c}$ and is such that $\mathfrak{h} / \mathfrak{c}=$ $\tau^{\mathrm{fg}, T}(\bar{R})$, the finitistic $T$-test ideal of $\bar{R}$, then $\mathfrak{h} \in \mathcal{I}(\Phi(E))$.

(ii) In particular, if $\mathfrak{h}^{\prime}$ denotes the unique ideal of $R$ that contains $\mathfrak{c}$ and is such that $\mathfrak{h}^{\prime} / \mathfrak{c}=\tau(\bar{R})$, the test ideal of $\bar{R}$, then $\mathfrak{h}^{\prime} \in \mathcal{I}(\Phi(E))$. 
(iii) If $\mathfrak{g}$ denotes the unique ideal of $R$ that contains $\mathfrak{c}$ and is such that $\mathfrak{g} / \mathfrak{c}=$ $\tau^{T}(\bar{R})$, the $T$-test ideal of $\bar{R}$, then $\mathfrak{g} \in \mathcal{I}(\Phi(E))$.

(iv) In particular, if $\mathfrak{g}^{\prime}$ denotes the unique ideal of $R$ that contains $\mathfrak{c}$ and is such that $\mathfrak{g}^{\prime} / \mathfrak{c}=\widetilde{\tau}(\bar{R})$, the big test ideal of $\bar{R}$, then $\mathfrak{g}^{\prime} \in \mathcal{I}(\Phi(E))$.

Proof. Straightforward modifications of the arguments given in the proof of [14, Corollary 3.2] will provide a proof for this.

2.9. Lemma. Assume that $(R, \mathfrak{m})$ is local, F-pure and a homomorphic image of an excellent regular local ring of characteristic $p$.

(i) There is a strictly ascending chain $0=\tau_{0} \subset \tau_{1} \subset \cdots \subset \tau_{t} \subset \tau_{t+1}=R$ of radical ideals of $R$ such that, for each $i=0, \ldots, t$, the reduced local ring $R / \tau_{i}$ is $F$-pure and its test ideal is $\tau_{i+1} / \tau_{i}$. We call this the test ideal chain of $R$. All of $\tau_{0}=0, \tau_{1}, \cdots, \tau_{t}$, and all their associated primes, belong to $\mathcal{I}(\Phi(E))$.

(ii) There is a strictly ascending chain $0=\widetilde{\tau}_{0} \subset \widetilde{\tau}_{1} \subset \cdots \subset \widetilde{\tau}_{w} \subset \widetilde{\tau}_{w+1}=R$ of radical ideals in $\mathcal{I}(\Phi(E))$ such that, for each $i=0, \ldots, w$, the reduced local ring $R / \widetilde{\tau}_{i}$ is F-pure and its big test ideal is $\widetilde{\tau}_{i+1} / \widetilde{\tau}_{i}$. We call this the big test ideal chain of $R$. All of $\widetilde{\tau}_{0}=0, \widetilde{\tau}_{1}, \cdots, \widetilde{\tau}_{w}$, and all their associated primes, belong to $\mathcal{I}(\Phi(E))$.

Note. In the case when $R$ is an ( $F$-pure) homomorphic image of an $F$-finite regular local ring, part (i) of this result is known and due to Janet Cowden Vassilev [16, §3].

Proof. (i) Set $\tau_{1}:=\tau(R)$, and note that $\tau(R) \in \mathcal{I}(\Phi(E))$. If $\tau_{1} \neq R$, apply Theorem 2.7 with the choice $\mathfrak{c}=\tau(R)=\tau_{1}$. That shows that $R / \tau_{1}$ is $F$-pure. Now argue by induction on $\operatorname{dim} R$, noting that $R / \tau_{1}$ is a homomorphic image of an excellent regular local ring of characteristic $p$. Use Theorem 2.7(v) to show that all of $\tau_{0}, \tau_{1}, \ldots, \tau_{t}$ belong to $\mathcal{I}(\Phi(E))$.

(ii) This is proved similarly.

\section{The F-finite case}

In the $F$-finite case, the results above have strong connections with work of $\mathrm{K}$. Schwede in [10], and the purpose of this section is to explore some of those connections. The introduction contains a description of certain properties of the set of all uniformly $F$-compatible ideals in an $F$-finite, $F$-pure local ring $R$, and some of these are similar to properties of the set of all fully $\Phi(E)$-special ideals of $R$ : we shall show in this section that, in this special case, an ideal of $R$ is uniformly $F$-compatible if and only if it is $\Phi(E)$-special, and that this is the case if and only if it is fully $\Phi(E)$-special.

3.1. Definition. Suppose that $R$ is $F$-finite; let $\mathfrak{b}$ be an ideal of $R$. Then $\mathfrak{b}$ is said to be uniformly $F$-compatible if, for every $n>0$ and every $\phi \in \operatorname{Hom}_{R}\left(R^{(n)}, R\right)$, we have $\phi\left(\mathfrak{b}^{(n)}\right) \subseteq \mathfrak{b}$.

3.2. Proposition (Schwede [10, Lemma 5.1]). Suppose that $(R, \mathfrak{m})$ is F-finite; let $\mathfrak{b}$ be an ideal of $R$. Then $\mathfrak{b}$ is uniformly $F$-compatible if and only if $\left(0::_{E} \mathfrak{b}\right) \subseteq$ $\left(\operatorname{ann}_{\Phi(E)}(\mathfrak{b} R[x, f])\right)_{0}$.

Thus when $R$ is $F$-finite and $F$-pure, $\mathfrak{b}$ is uniformly $F$-compatible if and only if it is fully $\Phi(E)$-special. 
Proof. Let $n \in \mathbb{N}$ and $r \in R$. Multiplication by $r$ yields an $R$-homomorphism of $R^{(n)}$, which, strictly speaking, we should denote by $r \operatorname{Id}_{R^{(n)}}$. Also $f^{n}: R \longrightarrow R^{(n)}$ is an $R$-homomorphism. Thus we can consider the composition of $R$-homomorphisms $R \stackrel{f^{n}}{\longrightarrow} R^{(n)} \stackrel{r}{\longrightarrow} R^{(n)}$.

Application of the functor $\bullet \otimes_{R} E$ yields a composition of $R$-homomorphisms

$$
R \otimes_{R} E \longrightarrow R^{(n)} \otimes_{R} E \stackrel{r}{\longrightarrow} R^{(n)} \otimes_{R} E,
$$

where the ' $r$ ' over the second arrow is an abbreviation for $r \operatorname{Id}_{R^{(n)}} \otimes_{R} E$. But $R^{(n)} \cong$ $R x^{n}$ as $(R, R)$-bimodules; furthermore, $\left(0:_{E} \mathfrak{b}\right) \cong \operatorname{Hom}_{R}(R / \mathfrak{b}, E)$. It follows that $\left(0::_{E} \mathfrak{b}\right) \subseteq\left(\operatorname{ann}_{\Phi(E)}(\mathfrak{b} R[x, f])\right)_{0}$ if and only if, for all $n \in \mathbb{N}$ and all $r \in \mathfrak{b}$, the composition

$$
\left(0::_{E} \mathfrak{b}\right) \stackrel{\subseteq}{\longrightarrow} E \stackrel{\cong}{\longrightarrow} R \otimes_{R} E \longrightarrow R^{(n)} \otimes_{R} E \stackrel{r}{\longrightarrow} R^{(n)} \otimes_{R} E
$$

(in which the second map is the natural isomorphism) is zero.

Let $M$ be an $R$-module. Recall that there is an $R$-homomorphism

$$
\xi_{M}: M \otimes_{R} E \longrightarrow \operatorname{Hom}_{R}\left(\operatorname{Hom}_{R}(M, R), E\right)
$$

such that, for $m \in M, e \in E$ and $g \in \operatorname{Hom}_{R}(M, R)$, we have $\left(\xi_{M}(m \otimes e)\right)(g)=g(m) e$. Furthermore, as $M$ varies, the $\xi_{M}$ constitute a natural transformation of functors; also $\xi_{M}$ is an isomorphism whenever $M$ is finitely generated. We shall use $D$ to denote the functor $\operatorname{Hom}_{R}(\bullet, E)$.

Since $R^{(n)}$ is a finitely generated $R$-module, $\left(0:_{E} \mathfrak{b}\right) \subseteq\left(\operatorname{ann}_{\Phi(E)}(\mathfrak{b} R[x, f])\right)_{0}$ if and only if, for all $n \in \mathbb{N}$ and all $r \in \mathfrak{b}$, the composition

$$
D(R / \mathfrak{b}) \rightarrow D(R) \stackrel{\cong}{\rightarrow} D\left(\operatorname{Hom}_{R}(R, R)\right) \rightarrow D\left(\operatorname{Hom}_{R}\left(R^{(n)}, R\right)\right) \stackrel{r}{\rightarrow} D\left(\operatorname{Hom}_{R}\left(R^{(n)}, R\right)\right)
$$

is zero. (Here, the first map is induced from the natural epimorphism $R \longrightarrow R / \mathfrak{b}$, the second map is the natural isomorphism, and the sequence from the middle term rightwards is the result of application of the functor $\operatorname{Hom}_{R}\left(\operatorname{Hom}_{R}(\bullet, R), E\right)$ to the composition $R \stackrel{f^{n}}{\longrightarrow} R^{(n)} \stackrel{r}{\longrightarrow} R^{(n)}$ described at the beginning of the proof.)

Since $D$ is a faithful functor (because $E$ is an injective cogenerator for $R$ ), we can deduce that $\left(0:_{E} \mathfrak{b}\right) \subseteq\left(\operatorname{ann}_{\Phi(E)}(\mathfrak{b} R[x, f])\right)_{0}$ if and only if, for all $n \in \mathbb{N}$ and all $r \in \mathfrak{b}$, the composition

$$
\operatorname{Hom}_{R}\left(R^{(n)}, R\right) \stackrel{r}{\longrightarrow} \operatorname{Hom}_{R}\left(R^{(n)}, R\right) \longrightarrow \operatorname{Hom}_{R}(R, R) \stackrel{\cong}{\longrightarrow} R \longrightarrow R / \mathfrak{b}
$$

is zero, that is, if and only if $\mathfrak{b}$ is uniformly $F$-compatible.

3.3. Proposition (Schwede [10]). Suppose that $(R, \mathfrak{m})$ is F-finite, and let $\mathfrak{a}$ be an ideal of $R$. Note that the completion $\widehat{R}$ of $R$ is again $F$-finite.

(i) If $\mathfrak{a}$ is a uniformly $F$-compatible ideal of $R$, then $\mathfrak{a} \widehat{R}$ is a uniformly $F$ compatible ideal of $\widehat{R}$. See Schwede [10, Lemma 3.9].

(ii) If $\mathfrak{C}$ is a uniformly $F$-compatible ideal of $\widehat{R}$, then $\mathfrak{C} \cap R$ is a uniformly $F$ compatible ideal of $R$. See Schwede [10, Lemma 3.8].

Proof. For a finitely generated $R$-module $M$, we identify $\widehat{M}$ with $M \otimes_{R} \widehat{R}$ in the usual way, and we note that there is a natural $\widehat{R}$-isomorphism $\psi_{M}: \operatorname{Hom}_{R}(M, R) \otimes_{R} \widehat{R} \cong$ $\operatorname{Hom}_{\widehat{R}}\left(M \otimes_{R} \widehat{R}, R \otimes_{R} \widehat{R}\right)$ for which $\psi_{M}(g \otimes \widehat{r})=\widehat{r}\left(g \otimes \operatorname{Id}_{\widehat{R}}\right)$ for all $g \in \operatorname{Hom}_{R}(M, R)$ 
and $\widehat{r} \in \widehat{R}$. Let $n \in \mathbb{N}$. Consideration of Cauchy sequences shows that $\widehat{M^{(n)}}=\widehat{M}^{(n)}$. In particular, $\widehat{R^{(n)}}=\widehat{R}^{(n)}$ and $\widehat{\mathfrak{a}^{(n)}}=(\widehat{\mathfrak{a}})^{(n)}=(\mathfrak{a} \widehat{R})^{(n)}$.

There is an $\widehat{R}$-isomorphism $\gamma: R^{(n)} \otimes_{R} \widehat{R} \cong \widehat{R}^{(n)}$ which maps $\mathfrak{a}^{(n)} \otimes_{R} \widehat{R}$ onto $(\mathfrak{a} \widehat{R})^{(n)}$. Also, the natural $\widehat{R}$-isomorphism $\delta: R \otimes_{R} \widehat{R} \cong \widehat{R}$ maps $\mathfrak{a} \otimes_{R} \widehat{R}$ onto $\mathfrak{a} \widehat{R}$.

(i) Let $\theta \in \operatorname{Hom}_{\widehat{R}}\left(R^{(n)} \otimes_{R} \widehat{R}, R \otimes_{R} \widehat{R}\right)$. By the above, there exist $\phi_{1}, \ldots, \phi_{t} \in$ $\operatorname{Hom}_{R}\left(R^{(n)}, R\right)$ and $\widehat{r}_{1}, \ldots, \widehat{r}_{t} \in \widehat{R}$ such that $\theta=\widehat{r}_{1}\left(\phi_{1} \otimes \operatorname{Id}_{\widehat{R}}\right)+\cdots+\widehat{r}_{t}\left(\phi_{t} \otimes \operatorname{Id}_{\widehat{R}}\right)$. Since $\phi_{i}\left(\mathfrak{a}^{(n)}\right) \subseteq \mathfrak{a}$ for all $n \in \mathbb{N}$ and $i=1, \ldots, t$, we see that $\theta\left(\mathfrak{a}^{(n)} \otimes_{R} \widehat{R}\right) \subseteq \mathfrak{a} \otimes_{R} \widehat{R}$ for all $n \in \mathbb{N}$. Use of the above-mentioned isomorphisms $\gamma$ and $\delta$ now enables us to conclude that $\mathfrak{a} \widehat{R}$ is a uniformly $F$-compatible ideal of $\widehat{R}$.

(ii) Let $\phi \in \operatorname{Hom}_{R}\left(R^{(n)}, R\right)$, and set $\mathfrak{c}:=\mathfrak{C} \cap R$. Then

$$
\phi \otimes \operatorname{Id}_{\widehat{R}} \in \operatorname{Hom}_{\widehat{R}}\left(R^{(n)} \otimes_{R} \widehat{R}, R \otimes_{R} \widehat{R}\right)
$$

and $\delta \circ\left(\phi \otimes \operatorname{Id}_{\widehat{R}}\right) \circ \gamma^{-1}$ maps $\mathfrak{C}^{(n)}$ into $\mathfrak{C}$, and therefore maps $(\mathfrak{c} \widehat{R})^{(n)}$ into $\mathfrak{C}$. Therefore $\delta \circ\left(\phi \otimes \operatorname{Id}_{\widehat{R}}\right)$ maps $\mathfrak{c}^{(n)} \otimes_{R} \widehat{R}$ into $\mathfrak{C}$, so that $\phi(a) \in \mathfrak{C} \cap R=\mathfrak{c}$ for all $a \in \mathfrak{c}^{(n)}$. Therefore $\mathfrak{c}$ is a uniformly $F$-compatible ideal of $R$.

3.4. Theorem. Suppose that $(R, \mathfrak{m})$ is F-pure and F-finite. Then each $\Phi(E)$-special ideal $\mathfrak{a}$ of $R$ is automatically fully $\Phi(E)$-special.

Proof. Note that $\widehat{R}$ is also $F$-pure, by Hochster and Roberts [3, Corollary 6.13]. Also, $\widehat{R}$ is $F$-finite, because the completion of the finitely generated $R$-module $R^{(1)}$ is $\widehat{R}^{(1)}$.

Thus, by definition, $\mathfrak{a}$ is the $R$-annihilator of an $R[x, f]$-submodule of $\Phi(E)$. It follows from Lemma 1.11 that $\mathfrak{a}=\mathfrak{A} \cap R$ for some ideal $\mathfrak{A}$ of $\widehat{R}$ that is the $\widehat{R}$ annihilator of an $\widehat{R}[x, f]$-submodule of $\Phi_{\widehat{R}}(E)$. Thus $\mathfrak{A}$ is $\Phi_{\widehat{R}}(E)$-special. It follows from Proposition [1.7 that $\mathfrak{A}$ is a fully $\Phi_{\widehat{R}}(E)$-special ideal of $\widehat{R}$, and so is uniformly $F$-compatible, by Proposition [3.2. Therefore, by Proposition 3.3(ii), the contraction $\mathfrak{A} \cap R=\mathfrak{a}$ is a uniformly $F$-compatible ideal of $R$, and is therefore fully $\Phi(E)$-special, by Proposition 3.2 again.

3.5. Corollary. Suppose that $(R, \mathfrak{m})$ is F-pure and F-finite; let $\mathfrak{a}$ be an ideal of $R$. Then the following statements are equivalent:

(i) a is uniformly F-compatible;

(ii) $\mathfrak{a}$ is $\Phi(E)$-special;

(iii) $\mathfrak{a}$ is fully $\Phi(E)$-special.

Proof. This is now immediate from Proposition 3.2 and Theorem 3.4

3.6. Question. Suppose that $(R, \mathfrak{m})$ is $F$-pure.

We have seen that each $\Phi(E)$-special ideal of $R$ is fully $\Phi(E)$-special if $R$ is complete (by Proposition 1.7) or if $R$ is a homomorphic image of an excellent regular local ring of characteristic $p$ (by Theorem 2.3) or if $R$ is $F$-finite (by Theorem 3.4).

Note that each complete local ring is excellent, and that each $F$-finite local ring of characteristic $p$ is excellent (by E. Kunz [4, Theorem 2.5]). The above results raise the following question. If the $F$-pure local ring $R$ is excellent, is it the case that every $\Phi(E)$-special ideal of $R$ is fully $\Phi(E)$-special? 


\section{A generalization of Aberbach's and Enescu's splitting prime}

Recall from [6, Remark 2.8 and Proposition 2.9] that G. Lyubeznik and K. E. Smith defined $(R, \mathfrak{m})$ to be strongly $F$-regular (even in the case where $R$ is not $F$ finite) precisely when the zero submodule of $E$ is tightly closed in $E$. See M. Hochster and C. Huneke [2, $\S 8]$.

4.1. Theorem. Suppose that $(R, \mathfrak{m})$ is $F$-pure and that every $\Phi(E)$-special ideal of $R$ is fully $\Phi(E)$-special. (For example, by Theorem 2.3, this would be the case if $R$ were a homomorphic image of an excellent regular local ring of characteristic $p$; it would also be the case if $R$ were F-finite, by Theorem 3.4.)

(i) There exists a unique largest $\Phi(E)$-special proper ideal, $\mathfrak{c}$ say, of $R$ and this is prime. Furthermore, $R / \mathfrak{c}$ is strongly $F$-regular.

(ii) Let $T$ be the $R[x, f]$-submodule of $\Phi(E)$ generated by $\left(0:_{E} \mathfrak{m}\right) \subseteq R \otimes_{R} E$. Then gr-ann $\operatorname{Rix,f]} T=\mathfrak{c} R[x, f]$.

Proof. (i) By Corollary 1.10, there is a unique largest $\Phi(E)$-special proper ideal $\mathfrak{c}$ of $R$, and this is prime. By Corollary 2.8(iv), the big test ideal of $R / \mathfrak{c}$ is $R / \mathfrak{c}$ itself, so that $1_{R / \mathfrak{c}}$ is a big test element for $R / \mathfrak{c}$. Therefore the zero submodule of $E_{R / \mathfrak{c}}(R / \mathfrak{m})$ is tightly closed in $E_{R / \mathfrak{c}}(R / \mathfrak{m})$, and so $R / \mathfrak{c}$ is strongly $F$-regular.

(ii) Note that $T$ is the image of the $R[x, f]$-homomorphism

$$
R[x, f] \otimes_{R}\left(0::_{E} \mathfrak{m}\right) \longrightarrow R[x, f] \otimes_{R} E=\Phi(E)
$$

induced by the inclusion map $\left(0:_{E} \mathfrak{m}\right) \stackrel{\subseteq}{\longrightarrow} E$. Let $\mathfrak{d}$ be the $\Phi(E)$-special ideal of $R$ for which gr-ann $\operatorname{anx}_{R, f]} T=\mathfrak{d} R[x, f]$. Since $\mathfrak{d}$ annihilates $\left(0:_{E} \mathfrak{m}\right)$, we see that $\mathfrak{d}$ is proper. Suppose that there exists $\mathfrak{h} \in \mathcal{I}(\Phi(E)$ ) such that $\mathfrak{d} \subset \mathfrak{h} \subseteq \mathfrak{m}$. (The symbol ' $\subset$ ' is reserved to denote strict inclusion.) Thus we have $\left(0:_{E} \mathfrak{m}\right) \subseteq\left(0:_{E} \mathfrak{h}\right) \subseteq\left(0:_{E} \mathfrak{d}\right)$. But we know that every $\Phi(E)$-special ideal of $R$ is fully $\Phi(E)$-special, and therefore $\left(0::_{E} \mathfrak{h}\right) \subseteq\left(\operatorname{ann}_{\Phi(E)}(\mathfrak{h} R[x, f])\right)_{0}$. Since $\operatorname{ann}_{\Phi(E)}(\mathfrak{h} R[x, f])$ is an $R[x, f]$-submodule of $\Phi(E)$, it follows that

$$
T \subseteq \operatorname{ann}_{\Phi(E)}(\mathfrak{h} R[x, f]) \subseteq \operatorname{ann}_{\Phi(E)}(\mathfrak{d} R[x, f]) .
$$

Now take graded annihilators: in view of the bijective correspondence between the sets $\mathcal{I}(\Phi(E))$ and $\mathcal{A}(\Phi(E))$ alluded to in the Introduction, we have

$$
\begin{aligned}
& \mathfrak{d} R[x, f]=\operatorname{gr}_{-a n n} \operatorname{and}_{[x]}\left(\operatorname{ann}_{\Phi(E)}(\mathfrak{d} R[x, f])\right) \\
& \subseteq \operatorname{gr}^{-\operatorname{ann}_{R[x, f]}}\left(\operatorname{ann}_{\Phi(E)}(\mathfrak{h} R[x, f])\right)=\mathfrak{h} R[x, f] \\
& \subseteq \mathrm{gr}^{-\mathrm{ann}_{R[x, f]}} \mathrm{T}=\mathfrak{d} R[x, f] .
\end{aligned}
$$

Hence $\mathfrak{h}=\mathfrak{d}$ and we have a contradiction.

Thus $\mathfrak{d}$ is a maximal member of the set of proper $\Phi(E)$-special ideals of $R$; therefore $\mathfrak{d}=\mathfrak{c}$.

4.2. Definition (I. M. Aberbach and F. Enescu [1, Definition 3.2]). Suppose $(R, \mathfrak{m})$ is $F$-finite and reduced. Let $u$ be a generator for the socle $\left(0:_{E} \mathfrak{m}\right)$ of $E$. Aberbach and Enescu defined

$$
\mathfrak{P}=\left\{r \in R: r \otimes u=0 \text { in } R^{(n)} \otimes_{R} E \text { for all } n \gg 0\right\},
$$

an ideal of $R$. 
In [1, §3], Aberbach and Enescu showed that in the case where $(R, \mathfrak{m})$ is $F$-finite and $F$-pure, and with the notation of 4.2 , the ideal $\mathfrak{P}$ is prime and is equal to the set of elements $c \in R$ for which, for all $e \in \mathbb{N}$, the $R$-homomorphism $\phi_{c, e}: R \longrightarrow R^{1 / p^{e}}$ for which $\phi_{c, e}(1)=c^{1 / p^{e}}$ does not split over $R$. Aberbach and Enescu call this $\mathfrak{P}$ the splitting prime for $R$. By [1, Theorem 4.8(i)], the $\operatorname{ring} R / \mathfrak{P}$ is strongly $F$-regular.

4.3. Proposition. Suppose that $(R, \mathfrak{m})$ is F-finite and F-pure. Let $\mathfrak{P}$ be Aberbach's and Enescu's splitting prime, as in 4.2. Let $\mathfrak{q}$ be the unique largest $\Phi(E)$-special proper ideal of $R$, as in Theorem 4.1. Then $\mathfrak{P}=\mathfrak{q}$.

Proof. Let $u$ be a generator for the socle $\left(0:_{E} \mathfrak{m}\right)$ of $E$. We can write

$$
\mathfrak{P}=\left\{r \in R: r x^{n} \otimes u=0 \text { in } R x^{n} \otimes_{R} E \text { for all } n \gg 0\right\} .
$$

Now for a positive integer $j$ and $r \in R$, if $r x^{j} \otimes u=0$ in $\Phi(E)$, then $x\left(r x^{j-1} \otimes u\right)=$ $r^{p} x^{j} \otimes u=0$, so that $r x^{j-1} \otimes u=0$ because the left $R[x, f]$-module $\Phi(E)$ is $x$-torsionfree. Therefore

$$
\mathfrak{P}=\left\{r \in R: r x^{n} \otimes u=0 \text { in } R x^{n} \otimes_{R} E \text { for all } n \geq 0\right\} .
$$

Let $T$ be the $R[x, f]$-submodule of $\Phi(E)$ generated by $\left(0:_{E} \mathfrak{m}\right) \subseteq R \otimes_{R} E$. We thus see that $\mathfrak{P} R[x, f]=\operatorname{gr}^{-a n n n_{R[x, f]}} T$, and this is $\mathfrak{q} R[x, f]$ by Theorem 4.1 . Hence $\mathfrak{P}=\mathfrak{q}$.

4.4. Remarks. Suppose that $(R, \mathfrak{m})$ is $F$-pure and a homomorphic image of an excellent regular local ring $S$ of characteristic $p$ modulo an ideal $\mathfrak{A}$. By Theorem 4.1(i), there exists a unique largest $\Phi(E)$-special proper ideal, $\mathfrak{q}$ say, of $R$ and this is prime. Let $\mathfrak{Q}$ be the unique ideal of $S$ containing $\mathfrak{A}$ for which $\mathfrak{Q} / \mathfrak{A}=\mathfrak{q}$.

(i) The results of this section suggest that $\mathfrak{q}$ can be viewed as a generalization of Aberbach's and Enescu's splitting prime: for example, Proposition 4.3 shows that $\mathfrak{q}$ is that splitting prime in the case where $R$ is, in addition, $F$-finite.

(ii) Note that $R / \mathfrak{q}$ is strongly $F$-regular (in the sense of Lyubeznik and Smith mentioned at the beginning of the section).

(iii) By Proposition $\left[2.2\right.$, we have $\left(\mathfrak{A}^{\left[p^{n}\right]}: \mathfrak{A}\right) \subseteq\left(\mathfrak{Q}^{\left[p^{n}\right]}: \mathfrak{Q}\right)$ for all $n \in \mathbb{N}$. In the special case in which $S$ is $F$-finite, this result was obtained by Aberbach and Enescu [1, Proposition 4.4].

\section{REFERENCES}

[1] I. M. Aberbach and F. Enescu, 'The structure of F-pure rings', Math. Z. 250 (2005) 791-806.

[2] M. Hochster and C. Huneke, 'Tight closure, invariant theory and the Briançon-Skoda Theorem', J. Amer. Math. Soc. 3 (1990) 31-116.

[3] M. Hochster and J. L. Roberts, 'Rings of invariants of reductive groups acting on regular rings are Cohen-Macaulay', Advances in Math. 13 (1974) 115-175.

[4] E. Kunz, 'On Noetherian rings of characteristic p', American J. Math. 98 (1976) 999-1013.

[5] G. Lyubeznik, ' $F$-modules: applications to local cohomology and $D$-modules in characteristic $p>0$, J. reine angew. Math. 491 (1997) 65-130.

[6] G. Lyubeznik and K. E. Smith, 'On the commutation of the test ideal with localization and completion', Transactions Amer. Math. Soc. 353 (2001) 3149-3180.

[7] D. Popescu, 'General Néron desingularization', Nagoya Math. J. 100 (1985) 97-126.

[8] D. Popescu, 'General Néron desingularization and approximation', Nagoya Math. J. 104 (1986) 85-115. 
[9] N. Radu, 'Une classe d'anneaux Noétheriens', Rev. Roumaine Math. Pures Appl. 37 (1992) $79-82$.

[10] K. Schwede, 'Centers of F-purity', Math. Z. 265 (2010) 687-714.

[11] R. Y. Sharp, 'Graded annihilators of modules over the Frobenius skew polynomial ring, and tight closure', Transactions Amer. Math. Soc. 359 (2007) 4237-4258.

[12] R. Y. Sharp, 'An excellent $F$-pure ring of prime characteristic has a big tight closure test element', Transactions Amer. Math. Soc. 362 (2010) 5455-5481.

[13] R. Y. Sharp, 'Big tight closure test elements for some non-reduced excellent rings', J. Algebra 349 (2012) 284-316.

[14] R. Y. Sharp, 'Tight closure with respect to a multiplicatively closed subset of an $F$-pure local ring', J. Pure Appl. Algebra (2014), http://dx.doi.org/10.1016/j.jpaa.2014.05.021.

[15] D. W. Sharpe and P. VÁmos, Injective modules, Cambridge Tracts in Mathematics and Mathematical Physics 62 (Cambridge University Press, Cambridge, 1972).

[16] J. C. Vassilev, 'Test ideals in quotients of $F$-finite regular local rings', Transactions Amer. Math. Soc. 350 (1998) 4041-4051.

School of Mathematics and Statistics, University of Sheffield, Hicks Building, Sheffield S3 $7 R H$, United Kingdom

E-mail address: R.Y.Sharp@sheffield.ac.uk 